\title{
NON-HEADER IMPACT EXPOSURE AND KINEMATICS OF MALE YOUTH SOCCER PLAYERS
}

\author{
$\underline{\text { Declan A. Patton, }}{ }^{1}$ Colin M. Huber, ${ }^{1,2}$ Susan S. Margulies, ${ }^{3}$ Christina L. Master, ${ }^{1,4,5}$ and \\ Kristy B. Arbogast ${ }^{1,4}$ \\ ${ }^{1}$ Center for Injury Research and Prevention, Children's Hospital of Philadelphia, Philadelphia, PA \\ ${ }^{2}$ Department of Bioengineering, University of Pennsylvania, Philadelphia, PA, \\ ${ }^{3}$ Wallace H. Coulter Department of Biomedical Engineering, Georgia Institute of Technology and \\ Emory University, Atlanta, GA, \\ ${ }^{4}$ Perelman School of Medicine, University of Pennsylvania, Philadelphia, PA, \\ ${ }^{5}$ Sports Medicine and Performance Center, Children's Hospital of Philadelphia, Philadelphia, PA
}

Corresponding Author: Declan Patton

Email:PATTONDA@chop.edu

https://doi.org/10.34107/BiomedSciInstrum.57.04106

\begin{abstract}
Previous studies have investigated the head impact kinematics of purposeful heading in youth soccer; however, less than a third of all head injuries in youth soccer have been found to involve ball contact. The aim of the current study was to identify the head impact kinematics and exposure not associated with purposeful heading of the ball in male youth soccer. Headband-mounted sensors were used to monitor the head kinematics of male junior varsity and middle school teams during games. Video analysis of sensor-recorded events was used to code impact mechanism, surface and site. Junior varsity players had non-header impact rates of 0.28 per athlete-exposure (AE) and 0.37 per player-hour ( $\mathrm{PH}$ ), whereas middle school players had relatively lower non-header impact rates of 0.16 per AE and 0.25 per PH. Such impact rates fell within the large range of values reported by previous studies, which is likely affected by sensor type and recording trigger threshold. The most common non-header impact mechanism in junior varsity soccer was player contact, whereas ball-to-head was the most common non-header impact mechanism in middle school soccer. Non-header impacts for junior varsity players had median peak kinematics of $31.0 \mathrm{~g}$ and $17.4 \mathrm{rad} / \mathrm{s}$. Non-header impacts for middle school players had median peak kinematics of 40.6 $\mathrm{g}$ and $16.2 \mathrm{rad} / \mathrm{s}$. For non-header impacts, ball impacts to the rear of the head the highest peak kinematics recorded by the sensor. Such data provide targets for future efforts in injury prevention, such as officiating efforts to control player-to-player contact.
\end{abstract}

Keywords: biomechanics, concussion, head impact sensor, injury prevention, soccer

\section{INTRODUCTION}

The game of soccer is unique in that the head is used to purposefully redirect the path of the ball as part of an integral skill known as a "header". Non-header impacts also occur in soccer, which comprise falls, player contact and ball-to-head impacts. In contrast to headers, ball-to-head impacts are not purposefully performed by the player but are a result of the ball impacting the head of an unsuspecting player. Understanding the head impact burden of these non-header impacts is important to fully characterize the potential for head injuries in soccer.

Recent technological advances have enabled the development of head impact sensors to estimate the head impact exposure of human subjects in vivo [1]. Increasingly, research studies have used such technology to collect head impact data in sports [2]. Several previous studies have investigated the head impact kinematics of purposeful heading in youth soccer [3-7] and policies have been developed to minimize head impact exposure in youth soccer. For example, US Soccer eliminated heading for children under the age of 10 years and limited the amount of heading in training sessions for children between the ages of 11 and 13 years [8,9]. However, less than a third of all head injuries in youth soccer 